\title{
Fanconi anemia in twins with neutropenia: A case report
}

\author{
WENJUN DENG ${ }^{1 *}$, MINGYI ZHAO ${ }^{2 *}$, YINGTING LIU ${ }^{1}$, LIZHI CAO $^{1}$ and MINGHUA YANG ${ }^{1}$ \\ ${ }^{1}$ Department of Pediatrics, Xiangya Hospital, Central South University, Changsha, Hunan 410008; \\ ${ }^{2}$ Department of Pediatrics, The Third Xiangya Hospital, Central South University, Changsha, Hunan 410013, P.R. China
}

Received July 23, 2017; Accepted January 10, 2018

DOI: $10.3892 / \mathrm{ol} .2018 .9304$

\begin{abstract}
Fanconi anemia (FA) is a rare inherited disease caused by mutations in genes that are primarily involved in DNA damage response or repair. The disease is often characterized by congenital malformations, progressive bone marrow failure, abnormal skin pigmentation patterns and susceptibility to cancer. The present study describes a pair of 4-year-old male twins, both of whom had been suffering from upper respiratory tract infections for $>2$ years. There was no indication of discomfort including fever, coughing, bleeding or fatigue from either child when the upper respiratory tract infection disappeared. Physical examination of the twins did not reveal anything significant, and no external anomalies were observed. In order to obtain additional diagnostic evidence, next-generation gene sequencing, chromosome breakage analysis and comet assays were performed. The results revealed double heterozygous mutations in the Fanconi Anemia Complementation Group D2 gene of the twins, therefore providing a conclusive diagnosis of FA. The case highlights how difficulties in clinical diagnosis may be overcome by including genetic screening tests into the range of diagnostic tests, which may also reveal unexpected results.
\end{abstract}

\section{Introduction}

Neutropenia is a common abnormality in childhood with an incidence of $12.36 \%$ in Beijing from July 2015 to July 2016 (1), and is most often associated with infections, partcularly respiratory tract infections, skin soft tissue infections, and inherited bone marrow failure syndromes (IBMFS) is one of the causes of neutropenia. Fanconi anemia (FA), a rare autosomal recessive condition, is a hereditary IBMFS that was first described by Guido Fanconi in 1927 (2). The mortality

Correspondence to: Professor Minghua Yang, Department of Pediatrics, Xiangya Hospital, Central South University, 87 Xiangya Road, Changsha, Hunan 410008, P.R. China

E-mail: yangminghua@csu.edu.cn

*Contributed equally

Key words: twins, Fanconi anemia, neutropenia, Fanconi anemia complementation group D2, next-generation gene sequencing rate of FA in the United States and Israel is 1-5 cases for every 1 million people, with a carrier frequency of $\sim 1$ in 300 individuals (3). Although it is often characterized by congenital malformations, progressive bone marrow failure, abnormal skin pigmentation patterns and susceptibility to cancer (4), at least $25 \%$ of patients with FA have few or no abnormal symptoms that may aid in its early diagnosis (5). As a hereditary disease, at least 16 genes including Fanconi Anemia Complementation Group (FANC)A, FANCC, FANCG, FANCD1 and FANCD2 have been identified to cause FA. Among these, mutations in the FANCD1 and FANCD2 genes are the causative factor in $5 \%$ of all cases of this disease (6-12). The present case report describes a pair of twins whom suffered recurrent upper respiratory tract infections for $>2$ years prior to the study. They were identified to have FA, caused by a double heterozygous mutation of FANCD2.

\section{Case report}

The present study was approved by the Ethical Committee of Xiangya Hospital (Changsha, Hunan, China). Written informed consent was obtained from the parents of the twins.

A pair of 4-year-old male mono-twins who suffered a recurrent upper respiratory tract infection for $>2$ years presented to the pediatric outpatient ward of Xiangya Hospital, Central South University (Changsha, China) on September 15, 2015 , following a routine blood test that had been conducted on the twins (for a recurrent upper respiratory tract infection lasting $>2$ years) performed on the same day and had demonstrated abnormal results (the routine blood test for the elder brother revealed a white blood cell count (WBC) of 2.6x $10^{9} / 1$ (normal range, $4-9.5 \times 10^{9} / 1$ ), hemoglobin (HGB) of $118 \mathrm{~g} / 1$ (normal range, $120-175 \mathrm{~g} / \mathrm{l}$ ), platelet count (PLT) of $340 \times 10^{12} / 1$ (normal range, 125-350 $\times 10^{9} / 1$ ), and neutrophil granulocyte count (NE) of $0.7 \times 10^{9} / 1$ (normal range, $1.8-6.3 \times 10^{9} / 1$ ); the younger brother demonstrated a WBC of $2.2 \times 10^{9} / 1$, HGB of $110 \mathrm{~g} / 1$, PLT of $269 \times 10^{12} / 1$, and NE of $\left.0.8 \times 10^{9} / 1\right)$. There was no indication of discomfort from either child. The two patients were of normal height and weight according to age (13) and had no observable physical deformities, and physical examination did not reveal anything significant. Blood samples from the twins were tested repeatedly ( $>10$ times) every 1 to 2 months over the past 2 years, revealing WBC of $2.2 \times 10^{9}$ to $4.3 \times 10^{9} / 1$, HGB from 100 to $120 \mathrm{~g} / \mathrm{l}$, PLT from $210 \times 10^{12}$ to $314 \times 10^{12} / 1$ and $\mathrm{NE}$ from $0.7 \times 10^{9}$ to $1 \times 10^{9} / 1$. Other laboratory test results, including those for immunoglobulins ( $\operatorname{IgG}, \operatorname{Ig} \mathrm{A}, \operatorname{Ig}$ M and $\operatorname{IgE}$ ), 
lymphocyte subsets, virus antibodies (herpes simplex virus, respiratory syncytial virus, adenovirus, Epstein-Barr virus, coxsackievirus and cytomegalovirus), random blood glucose and endocrines (sex, thyroid and growth hormones), were not abnormal. Imaging analysis, including chest radiography, and cardiac and abdominal ultrasounds, did not reveal any areas of concern. Bone marrow cell morphology was assessed as follows: The punctured bone marrow fluid was placed at one end of a slide glass, and one end of a pushing piece with a flat edge was placed in front of the bone marrow fluid, and the bone marrow fluid was smoothly pushed forward to the other end of the slide glass, so that a thin layer of bone marrow fluid remained on the slide glass, then the slide was air-dried and fixed with methanol for $5 \mathrm{~min}$ at $25^{\circ} \mathrm{C}$. Next, the slide was dyed with Wright-Giemsa stain (Wright stain consisted of $0.1 \mathrm{~g}$ Wright's dye and $60 \mathrm{ml}$ methanol, and the Giemsa stain consisted of $0.5 \mathrm{~g}$ Giemsa's dye, $33 \mathrm{ml}$ methanol and $33 \mathrm{ml}$ pure glycerin, purchased from Beijing Solarbio Science \& Technology Co., Ltd., Beijing, China) for $5 \mathrm{~min}$ at $25^{\circ} \mathrm{C}$ prior to observation with optical microscope (magnification, $\mathrm{x} 100)$ to determine the bone marrow morphology and the degree of bone marrow hyperplasia. The bone marrow cell morphology test revealed a normal result. In order to obtain a more accurate diagnosis, next-generation sequencing (NGS) was performed. Genomic DNA samples obtained from $2 \mathrm{ml}$ peripheral blood were sheared by sonication. The sheared genomic DNA was then hybridized using the NimbleGen 2.0 probe sequence capture array (Roche Molecular Diagnostics, Pleasanton, CA, USA) to enrich exonic DNA (Joy Orient Translational Medicine Research Centre, Co., Ltd., Beijing, China). A $1 \mu \mathrm{g}$ DNA library was mixed with Buffer BL and GenCap gene panel probe (MyGenostics, Inc., Medford, MA, USA), heated at $95^{\circ} \mathrm{C}$ for $7 \mathrm{~min}$ and $65^{\circ} \mathrm{C}$ for $2 \mathrm{~min}$ on a polymerase chain reaction (PCR) machine. Subsequently, $23 \mu \mathrm{l}$ of the $65^{\circ} \mathrm{C}$ pre-warmed Buffer HY (MyGenostics, Inc.) was added to the mix, and the mixture was held at $65^{\circ} \mathrm{C}$ for $22 \mathrm{~h}$ for hybridization. MyOne beads (50 $\mu$ 1; Life Technology; Thermo Fisher Scientific, Inc., Waltham, MA, USA) were washed in $500 \mu \mathrm{l} 1 \mathrm{X}$ binding buffer 3 times and resuspended in $80 \mu \mathrm{l}$ $1 \mathrm{X}$ binding buffer. A total of $64 \mu 12 \mathrm{X}$ binding buffer was added to the hybrid mix and transferred to the tube with $80 \mu \mathrm{l}$ MyOne beads. The mix was rotated for $1 \mathrm{~h}$ at room temperature on a rotator. The beads were then washed with WB1 buffer at room temperature for 15 min once and WB3 buffer at $65^{\circ} \mathrm{C}$ for $15 \mathrm{~min} 3$ times. The bound DNA was then eluted using elution buffer $(1 \mathrm{~mol} / 1$ Tris- $\mathrm{HCl} 1 \mathrm{ml}$, and $0.5 \mathrm{~m}$ EDTA $0.2 \mathrm{ml}$ and $100 \mathrm{ml}$ distilled water). The eluted DNA was amplified for 15 cycles using the following program: $98^{\circ} \mathrm{C}$ for $30 \mathrm{sec}$ (1 cycle); $98^{\circ} \mathrm{C}$ for $25 \mathrm{sec}, 65^{\circ} \mathrm{CC}$ for $30 \mathrm{sec}, 72^{\circ} \mathrm{C}$ for $30 \mathrm{sec}$ ( 15 cycles); and $72^{\circ} \mathrm{C}$ for 5 min ( 1 cycle). The PCR product was purified using SPRI beads (Beckman Coulter, Inc., Brea, CA, USA) according to manufacturer's protocol. The libraries were first tested for size distribution and concentration using the Agilent Bioanalyzer 2100 with the 2100 Bioanalyzer Expert software (Agilent Technologies, Inc., Santa Clara, CA, USA). The samples were then sequenced on an Illumina Hiseq2500 with HiSeq Control software (HCS2.0; Illumina, Inc., San Diego, CA, USA) and analyzed using DNASTAR software (version 5.0; DNASTAR, Inc., Madison, WI, USA). A total of 2 parallel reactions were performed for each sample, and
Protein structure prediction software (v1.1, downloaded from J. Craig Venter Institute, La Jolla, CA, USA) was used to predict the functional effect of amino acid substitutions and indels. NGS revealed double heterozygous mutations in the FCND2 gene in the two patients (Tables I-III; Fig. 1). Additional diagnostic evidence obtained by chromosome breakage analysis. Chromosome culture was performed using the standardized method of the International Atomic Energy Agency 405 technical report (14). Mitomycin C (0, 50 and $100 \mu \mathrm{g} / \mathrm{l}$; Kyowa Hakko Bio Co., Ltd, Tokyo, Japan) was added after $24 \mathrm{~h}$ and cells were cultured for $48 \mathrm{~h}$ at $37^{\circ} \mathrm{C}$, harvested, added a potassium chloride hypotonic $(0.188 \%)$ solution for $20 \mathrm{~min}$ at $37^{\circ} \mathrm{C}$, pre-fixed with glacial acetic acid-methanol fixed fluid (consisting of glacial acetic acid and methanol at a ratio of 1:3) for $15 \mathrm{~min}$ at room temperature. A total of 1-2 drops was dropped onto slides from $40-50 \mathrm{~cm}$ and dried naturally prior to observation using a light microscope (magnification, $\mathrm{x} 100$ ). The number of chromosome aberration cells and the chromosome breakage number were counted under the microscope, and the chromosome aberration rate and breakage rate was analyzed. The breakage rate in 100 cells was defined as (the number of broken chromosomes/100) x100\%, aberration rate was defined as (the number of cells which appeared to exhibit broken chromosomes/100) x100\%. The chromosome aberration rate and breakage rate of the experimental group were considered to be positive if they were higher compared with that of the control group. A total of 100 mitotic images were analyzed per specimen. The results are presented in Table IV. A comet assay was also performed. Cytochalasin B $(6 \mu \mathrm{g} / \mathrm{ml}$, at $37^{\circ} \mathrm{C}$ for $28 \mathrm{~h}$ ), used to induce cell DNA damage, and ethidium bromide were purchased from Sigma-Aldrich (Merck KGaA, Darmstadt, Germany). A Nikon 90i fluorescence microscope was purchased from Nikon Corporation (Tokyo, Japan). The chromosome image analysis system GK-1303 was purchased from Leica Microsystems GmbH (Wetzlar, Germany) and the Sanyo MCO-20AIC CO2 incubator was purchased from SANYO Semiconductor Manufacturing Co., Ltd. (Sakata, Japan). The normal-melting-point agarose was obtained from Biowest USA (Riverside, MO, USA) and the low-melting-point agarose was from Promega Corporation (Madison, WI, USA). Tris- $\mathrm{HCl}$, dimethyl sulfoxide (DMSO) and Triton X-100 were purchased from Sigma-Aldrich, Merck KGaA. Lymphocyte-separated medium (lymphoprep) was purchased from Axis Shield Diagnostics, Ltd. (Dundee, UK). The horizontal-strip electrophoresis apparatus was from Bio-Rad Laboratories, Inc. (Hercules, CA, USA). The comet slides were from Bio-comet (Institute for Surgical Research and Hospital Management, Basel, Switzerland). The digital imaging system DMS300 was purchased from Leica Microsystems GmbH.

The comet assay was performed under neutral conditions, as described by Banath et al (15), but with a slight modification; specifically, special comet slides were used rather than general slides. There are gaps in comet slides to contain the agarose. Furthermore, less agarose was used in the procedure than originally described by Banath et al yielding a thinner gel agarose to enable clearer viewing under a fluorescence microscope. First, the comet slides was coated with $100 \mu 1$ normal-melting-point agarose $(0.075 \%)$; then, once the first agarose layer was coagulated, a mixture of $75 \mu \mathrm{l}$ low-melting-point agarose $(0.075 \%)$ and $25 \mu 1$ lymphocyte 
Table I. Mutation of FANCD2 in the patients.

Mutation information

\begin{tabular}{lllllll} 
& Chromosome location & Base change & AA change & MAF & Prediction & Disease \\
\hline FANCD2 & Chr3:10106532 & c.2141C $>\mathrm{T}(\mathrm{E} 23)$ & p.714, P $>\mathrm{L}$ & 0.085 & Harmful & Fanconi anemia \\
& Chr3:10130159 & p.3493 $>\mathrm{T}(\mathrm{E} 35)$ & p.1165, R $>\mathrm{W}$ & 0 & Harmful
\end{tabular}

FANCD2, Fanconi anemia complementation group 2; AA, amino acid; MAF, minor allele frequency.

Table II. Hereditary information of a FANCD2 allele (allele 1) of the patients.

\begin{tabular}{|c|c|c|c|c|c|}
\hline Family member & Gene & Mutation type & NA changes & AA changes & Prediction \\
\hline Twins & FCND2 & Heterozygous & C.2141C $>\mathrm{T}(\mathrm{E} 23)$ & p.P714L & Harmful \\
\hline Father & FCND2 & Normal & C.2141C (wild-type) & & Normal \\
\hline Mother & $F C N D 2$ & Heterozygous & C.2141C $>\mathrm{T}(\mathrm{E} 23)$ & p.P714L & Harmful \\
\hline
\end{tabular}

NA, Nucleic acid; AA, Amino acid; FANCD2, Fanconi anemia complementation group 2.

Table III. Hereditary information of a FANCD2 allele (allele 2) of the twins.

\begin{tabular}{llllll}
\hline Family member & Gene & Mutation type & NA changes & AA changes & Prediction \\
\hline Twins & $F C N D 2$ & Heterozygous & c.3493C>T(E35) & p.R1165W & Harmful \\
Father & $F C N D 2$ & Heterozygous & c.3493C>T(E35) & p.R1165W & Harmful \\
Mother & $F C N D 2$ & Normal & C.3493C (wild-type) & Normal \\
\hline
\end{tabular}

NA, nucleic acid; AA, amino acid; FANCD2, Fanconi anemia complementation group 2.
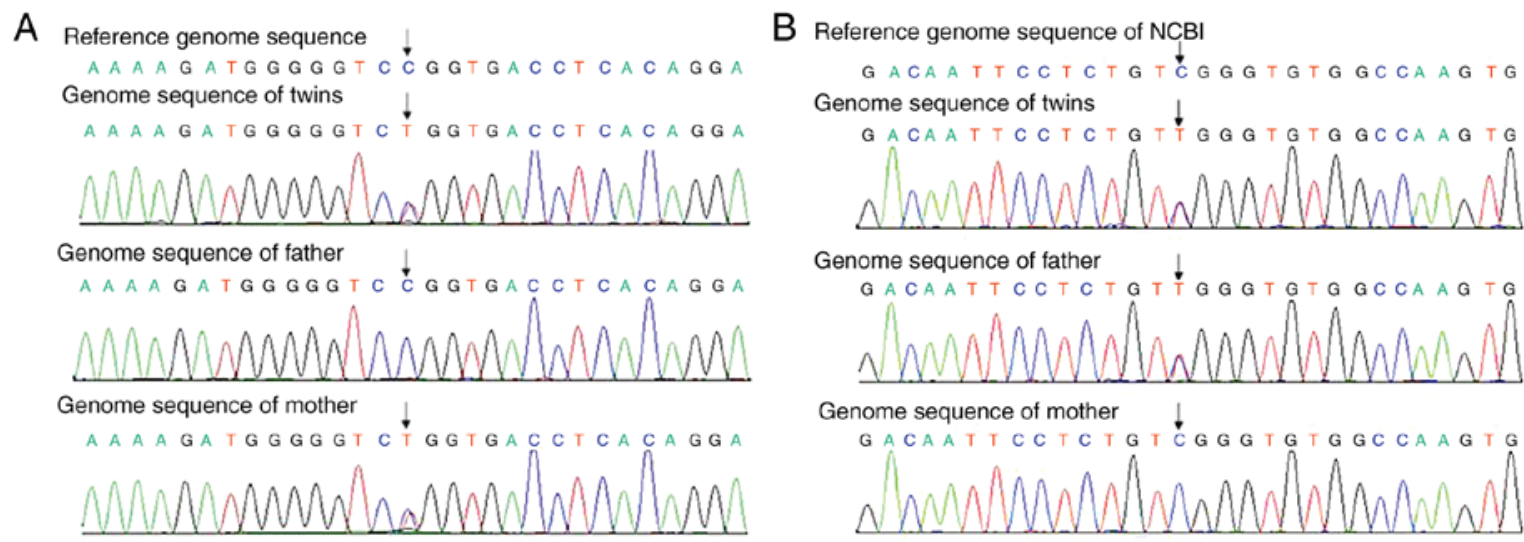

Figure 1. Fanconi Anemia Complementation Group D2 gene sequence diagram of the twin patients. (A) Genome sequence of Allele 1 for the patients and their parents. (B) Genome sequence of Allele 2 for the patients and their parents.

suspension was applied as the second layer. The comet slides were immersed in cold fresh lysis solution $(2.5 \mathrm{M} \mathrm{NaCl}, 1 \%$ $\mathrm{N}$-sodium lauryl sarcosinate, $30 \mathrm{mM} \mathrm{Na2EDTA,} 10 \mathrm{mM}$ Tris, $1 \%$ Triton X-100 and $10 \%$ DMSO) for $1.5 \mathrm{~h}$ at $4^{\circ} \mathrm{C}$. Following lysis, slides were placed in a horizontal electrophoresis tank pre-filled with cold fresh Tris-borate-EDTA (TBE, Medicago, Inc., Durham, NC, USA) buffer for $20 \mathrm{~min}$ at $4^{\circ} \mathrm{C}$ to loosen the tight double-helical structure of DNA for electrophoresis. Electrophoresis was then performed at $200 \mathrm{~mA}$ for $20 \mathrm{~min}$ in TBE buffer at room temperature. The slides were then rinsed twice with distilled water and were stained with ethidium bromide $(2 \mu \mathrm{g} / \mathrm{ml})$ for $10 \mathrm{~min}$ at $25^{\circ} \mathrm{C}$. All of the above procedures were performed in the dark to avoid supernumerary DNA damage. The comet slides were viewed using a Nikon 
Table IV. Chromosome breakage test.

A, Number of abberant cells per 100 cells .

\begin{tabular}{lccc}
\hline & \multicolumn{3}{c}{ MMC (ng/ml) } \\
\cline { 2 - 4 } Sample & 0 & 50 & 100 \\
\hline Twin 1 & 0.0 & $72.0 \pm 3.6$ & $90.0 \pm 1.7$ \\
Twin 2 & 0.0 & $70.5 \pm 1.8$ & $87.0 \pm 3.6$ \\
Control & $3.0 \pm 1.0$ & $18.6 \pm 2.1$ & $65.9 \pm 6.9$ \\
\hline
\end{tabular}

B, Number of breakage chromosomes per 100 cells.

\begin{tabular}{lccc}
\hline & \multicolumn{3}{c}{ MMC $(\mathrm{ng} / \mathrm{ml})$} \\
\cline { 2 - 4 } Sample & 0 & 50 & 100 \\
\hline Twin 1 & 0.0 & $172.0 \pm 6.0$ & $990.0 \pm 30.8$ \\
Twin 2 & 0.0 & $188.0 \pm 3.6$ & $964.0 \pm 15.1$ \\
Control & $3.0 \pm 0.6$ & $19.0 \pm 1.7$ & $114.8 \pm 5.3$ \\
\hline
\end{tabular}

MMC, mitomycin C.

90i fluorescence microscope (magnification, $\mathrm{x} 400$ ) and images of 100 comets were collected for each group using a digital imaging system (DMS300; Leica Microsystems GmbH). Cells that overlapped were not counted. All of the comet images were analyzed using Comet Assay Software Pect (cat. no., CASP 1.2.3 beta 1; CASPLab, University of Wroclaw, Institute of Theoretical Physics, Wroclaw, Poland), and the percentage of DNA in the comet tail, the tail length, the tail moment and the olive tail moment were recorded to characterize the lymphocytic DNA damage. Statistical analyses were performed using one-way analysis of variance and Dunnett's test with SPSS13.0 (SPSS Inc., Chicago, IL, USA), and P $<0.05$ was considered to indicate a statistically significant difference. The results, presented in Table V, revealed the Tail Moment of the comet experiment for twins were higher than that of the control group, which was statistically significant and therefore considered a positive result. Therefore, on the basis of these data, the twins were definitively diagnosed with FA.

\section{Discussion}

FA is a rare hereditary autosomal recessive disease caused by mutations in FA genes that are primarily involved in DNA damage response or repair (16). It is characterized by congenital malformations, progressive bone marrow failure, skin lesions and susceptibility to cancer (17). Early symptoms of FA include multiple malformations, including skeletal and ear deformities, renal malformation and abnormal skin pigmentation patterns, including café au lait spots and chromatosis (18). These are followed by progressive bone marrow failure, which begins to occur, on average, at 7 years of age (19), with the majority of patients eventually suffering from cancer (20). As these clinical manifestations lack specificity, it is often difficult to diagnose FA, particularly in patients who do not exhibit surface deformities or skin lesions. For this reason, tests including chromosome breakage analysis and comet assays are often performed to reach a definitive diagnosis. FA diagnosis is also confirmed by the subtyping of FA (i.e., determination of the complementation group) $(21,22)$. At present, sequencing of the FA genes has also become an important and accurate method of diagnosing the disease.

In the present case of the twins, the physical examination, necessary laboratory and imaging tests and lack of abnormal skin pigmentation patterns gave no indication of FA. Only changes in the levels of certain blood components were observed, without abnormal bone marrow cell morphology. Therefore, it was difficult to diagnose the cause of the abnormal blood results and even to select follow-up tests. Fortunately, their symptoms were similar to those of IBMFS, thereby providing a clue for the direction to follow. Next-generation gene sequencing was then performed and revealed that the two patients exhibited double heterozygous mutations in the FANCD2 gene, confirming that each of the parents was an obligate carrier. With this information, the twins were conclusively diagnosed with FA. In addition, the chromosome breakage experiment and comet assays demonstrated positive results, verifying the diagnosis.

The FANCD2 gene, located on chromosome 3 at position 3p25.3 (including 44 exons), codes for 2 isoforms of proteins (FANCD2-S and FANCD2-L) consisting of 1,451 amino acids, with molecular masses of 155 and $162 \mathrm{kDa}$, respectively. Ubiquitination of the FANCD2-encoded protein is of crucial importance in the pathogenesis of FA, as the ubiquitinated protein normally binds with Fanconi Anemia Complementation Group I and BRCA2, DNA Repair Associated, to repair damaged DNA. In the case of the two patients, next-generation sequencing revealed a c. $2141 \mathrm{C}>\mathrm{T}$ mutation in one of the alleles of FANCD2, termed Allele 1, which changes the 714th amino acid of this protein from a proline to a leucine. Protein structure prediction software (23) identified this change to be harmful. In addition, by testing this same FANCD2 site in the genome of the parents of the twins, it was identified that this mutation was passed on through the mother. In the other allele of FANCD2, termed Allele 2, a c.3493C $>$ T mutation was identified, which changes the 1,165 th amino acid of this protein from an arginine to a tryptophan. Protein structure prediction software also identified this change to be harmful. Testing of this site on the genomes of the parents revealed that this mutation originated from the father. On the basis of these results, it was concluded that harmful mutations in the two alleles of FANCD2 had been passed on to the twins, and they were definitively diagnosed with FA.

In summary, the following were the characteristics of the twins: Firstly, the two boys presented with the same symptoms during the early stages and appeared to be suffering from chronic illnesses for $>2$ years. Secondly, the twins indicated no discomfort upon hospital presentation and no positive results from the physical examination. Thirdly, specific abnormal blood test results and a positive comet assay result were observed, and double heterozygous mutations of FANCD2 were revealed. Fourthly, there were no other diseases aside from FA that accurately matched the symptoms and disease presentation of the twins. Therefore, on the basis of these results, the twins were diagnosed with FA. Analysis of the study may 
Table V. Comet assay.

\begin{tabular}{lcccccc}
\hline Group & Head DNA, \% & Tail DNA, \% & Tail length (pixel) & Comet length (pixel) & Tail moment & $\begin{array}{c}\text { Olive tail } \\
\text { moment }\end{array}$ \\
\hline Twin 1 & 94.39 & 5.61 & 13.33 & 65.11 & 0.79 & 0.51 \\
Twin 2 & 94.03 & 5.97 & 14.02 & 65.95 & 0.97 & 0.66 \\
Control & 98.02 & 1.98 & 8.92 & 64.03 & 0.50 & 0.60 \\
P-value for Twin 1 & $<0.001$ & $<0.001$ & $<0.001$ & $<0.001$ & $<0.001$ & 0.135 \\
P-value for Twin 2 & $<0.001$ & $<0.001$ & $<0.001$ & $<0.001$ & $<0.001$ & 0.112 \\
\hline
\end{tabular}

All comet images were analyzed using Comet Assay Software Project (cat. no., CASP 1.2.3 beta 1; CASPLab, University of Wroclaw, Institute of Theoretical Physics, Wroclaw, Poland) (29). Statistical analyses were performed using one-way analysis of variance by SPSS 13.0 (SPSS, Inc., Chicago, IL, USA), and $\mathrm{P}<0.05$ was considered to indicate a statistically significant difference.

cause the following to be questioned: Firstly, although the twins had abnormal routine blood test results, physical and imaging examinations revealed no malformations, and no abnormal skin pigmentation patterns were identified. The results of bone marrow morphology analysis were also identified to be normal, whereas the majority of cases of FA demonstrate all of the above symptoms. A possible explanation for this inconsistency is that the twins were in the early stage, or suffered from a mild subtype, of FA. Secondly, next-generation gene sequencing of FANCD2 does not appear to be sufficient to support the diagnosis of FA, as in the case of Allele 1. Although the protein structure prediction software predicted the change to be harmful, the minor allele frequency (MAF) for this mutation is $5.9 \%$ according to the Exome Aggregation Consortium (24). However, as the MAF for this mutation is as high as $5.9 \%$, this means that the homozygous mutation may be as high as $0.35 \%$, but as is not consistent with the incidence of FA, whether this type of mutation leads to FA is questionable. Our interpretation is that the present MAF value of Allele 1 is not representative of all existing data. An additional possible explanation is that these types of homozygous mutations, including that of Allele 1 in FA, result in mild cases FA or are not pathogenic, but when they exist together with a second mutation, for example a double heterozygous mutation, the symptoms become more marked. In addition, as the mutation of Allele 2 has not been identified previously for this gene, its MAF value is unknown and it may be a novel mutation. Nevertheless, based on protein structure prediction software, it was deduced that this mutation was also harmful. Unfortunately, the present study was not able to examine the protein structure and its mechanism.

It is well-known that hematopoietic stem cell transplantation (HSCT) is presently the only way to improve the hematopoietic environment and the long-term disease-free survival rate of patients with FA (25). Considering the fact that an early diagnosis of FA is difficult, and alongside the known problems of transplantation failure, graft-versus-host disease and numerous other transplantation-associated issues, there is no unified consensus on when to initiate HSCT for patients with FA (26). In a retrospective analysis of the prognosis of patients with FA who had undergone allogeneic HSCT, the European Group for Blood Marrow Transplantation identified that a patient age of $>10$ years with a history of $>20$ blood transfusions and hematopoietic system failure (absolute neutrophil count $<500 \times 10^{9} / 1$, platelet count $<20 \times 10^{9} / 1$, hemoglobin $<80 \mathrm{~g} / \mathrm{l}$ ) would be not conducive to the early reconstruction of the hematopoietic system (27). In the present case of the twin boys, due to their mild symptoms of tolerance according to the guidelines of FA (28), no additional tests or treatments were performed. The last out-patient follow-up time was December 28th, 2017, the twins had no symptoms at that time. The final routine blood tests revealed a WBC of $3.6 \times 10^{9} / 1$, HGB of $116 \mathrm{~g} / 1$, PLT $310 \times 10^{12} / 1$, and NE of $0.9 \times 10^{9} / 1$ for the elder brother, and a WBC of $3.0 \times 110^{9} / 1$, HGB of $112 \mathrm{~g} / 1$, PLT of $258 \times 10^{12} / 1$ and NE of $0.8 \times 10^{9} / 1$ for the younger brother. However, follow-up will be continued, and HSCT will be a viable treatment option for these patients in the future. Next-generation gene sequencing was performed for the parents, and they were provided with complete genetic information concerning FA, which may provide support in pre-implantation genetic diagnosis for future pregnancies if the couple wants to have another child.

Finally, it is necessary to state the reason the case of the present study was described, which was to share the results obtained. Firstly, the case was very rare, as each parent was a carrier for FA. Unfortunately, the illnesses of the twins were caused as a result of their double heterozygosity; however, it was notable that neither of them exhibited any typical symptoms of FA. Secondly, one novel mutation in the FADCN2 gene that may lead to FA was identified. Thirdly, the case demonstrated that difficulties in clinical diagnosis may be overcome by including genetic screening tests into the range of available diagnostic tests, which may also reveal unexpected results.

\section{Acknowledgements}

Not applicable.

\section{Funding}

The present study was supported by the National Natural Sciences Foundation of China (grant nos. 81570154, 81100359 and 81500231).

\section{Availability of data and materials}

The datasets generated and analyzed in the present study are included in this published article. 


\section{Authors' contributions}

WD and MZ performed the data analysis and wrote the manuscript. LC contributed to the conception of the study. YL contributed significantly to data analysis and manuscript preparation. MY helped perform the data analysis and conducted constructive discussions. All authors approved the final manuscript.

\section{Ethics and consent to participate}

The present study was approved by the Ethical Committee of Xiangya Hospital and written informed consent was obtained from the patient, or from the parent or guardians, as appropriate.

\section{Consent for publication}

Written informed consent was obtained for the publication of data and materials.

\section{Competing interests}

The authors declare that they have no competing interests.

\section{References}

1. Fang Ye, Li'nan Zhang, Jianli Sun, et al: Analysis of clinical features of leukopenia patients in a three level general hospital in Beijing. China Medical Herald 14: 60-63, 2017.

2. Crawford D and Dearmun A: Fanconi anaemia. Nurs Child Young People 29: 17, 2017.

3. Rosenberg PS, Tamary H and Alter BP: How high are carrier frequencies of rare recessive syndromes/contemporary estimates for Fanconi Anemia in the united states and Israe. Am J Med Genet A 155A: 1877-1883, 2011.

4. Pivovar A, Furquim CP, Bonfim C and Torres-Pereira CC: Mouth examination performance by children's parents and by adolescents in Fanconi Anemia. Pediatr Blood Cancer 64: doi: 10.1002/ pbc. 26622 .

5. Mohanty D: Understanding complexity of Fanconi anaemia. Indian J Med Res 143: 132-134, 2016

6. Cheung RS and Taniguchi T: Recent insights into the molecular basis of Fanconi Anemia: Genes, modifiers, and drivers. Int J Hematol 106: 335-344, 2017.

7. Fagerholm R, Sprott K, Heikkinen T, Bartkova J, Heikkilä P, Aittomäki K, Bartek J, Weaver D, Blomqvist C and Nevanlinna H: Overabundant FANCD2, alone and combined with NQO1, is a sensitive marker of adverse prognosis in breast cancer. Ann Oncol 24: 2780-2785, 2013.

8. de Winter JP, van der Weel L, de Groot J, Stone S, Waisfisz Q, Arwert F, Scheper RJ, Kruyt FA, Hoatlin ME and Joenje H: The Fanconi Anemia protein FANCF forms a nuclear complex with FANCA, FANCC and FANCG. Hum Mol Genet 9: 2665-2674, 2000.

9. Hess J, Unger K, Orth M, Schötz U, Schüttrumpf L, Zangen V, Gimenez-Aznar I, Michna A, Schneider L, Stamp R, et al: Genomic amplification of Fanconi Anemia complementation group A (FancA) in head and neck squamous cell carcinoma (HNSCC): Cellular mechanisms of radioresistance and clinical relevance. Cancer Lett 386: 87-99, 2017.

10. Svojgr K, Sumerauer D, Puchmajerova A, Vicha A, Hrusak O, Michalova K, Malis J, Smisek P, Kyncl M, Novotna D, et al: Fanconi Anemia with biallelic FANCD1/BRCA2 mutations Case report of a family with three affected children. Eur J Med Genet 59: 152-157, 2016.
11. Loizidou MA, Hadjisavvas A, Tanteles GA, Spanou-Aristidou E, Kyriacou K and Christophidou-Anastasiadou V: Fanconi Anemia-D1 due to homozygosity for the BRCA2 gene Cypriot founder mutation: A case report. Oncol Lett 11: 471-473, 2016.

12. Chen X, Bosques L, Sung P and Kupfer GM: A novel role for non-ubiquitinated FANCD2 in response to hydroxyurea-induced DNA damage. Oncogene 35: 22-34, 2016.

13. Coordinating Study Group of Nine Cities on Physical Growth and Development of Children; Capital Institute of Pediatrics: A national survey on growth of children under 7 years of age in nine cites of China, 2005. Zhonghua Er Ke Za Zhi 45: 609-614, 2007 (In Chinese).

14. International Atomic Energy Agency: Dicentric analysis[M]/Cytogenetic analysis for radiation dose assessment: A manual. Vienna: International Atomic Energy Agency, 2001: 27-59.

15. Banath JP, Fushiki M and Olive PL: Rejoining of DNA single-and double-strand breaks in human white blood cells exposed to ionizing radiation. Int J Radiat Biol 73: 649-660, 1998.

16. Burgos-Moron E, Calderon-Montano JM, Orta ML, Guillen-Mancina E, Mateos S and Lopez-Lazaro M: Cells Deficient in the Fanconi Anemia protein FANCD2 are hypersensitive to the cytotoxicity and DNA damage induced by coffee and caffeic acid. Toxins (Basel) 8: doi: 10.3390/toxins8070211.

17. D'Andrea AD: Susceptibility pathways in Fanconi's Anemia and breast cancer. N Engl J Med 362: 1909-1919, 2010.

18. Webb ML, Rosen H, Taghinia A, McCarty ER, Cerrato F, Upton J and Labow BI: Incidence of Fanconi Anemia in children with congenital thumb anomalies referred for diepoxybutane testing. J Hand Surg Am 36: 1052-1057, 2011.

19. Hanenberg H, Roellecke K and Wiek C: Stem cell genetic therapy for Fanconi Anemia - a New Hope. Curr Gene Ther 16: 309-320, 2017.

20. Rosenberg PS, Greene MH and Alter BP: Cancer incidence in persons with Fanconi Anemia. Blood 101: 822-826, 2003.

21. Aslan D, Ameziane N and De Winter JP: Molecular diagnosis of Fanconi Anemia with next-generation sequencing in a case with subtle signs and a negative chromosomal breakage test. Turk J Pediatr 57: 282-285, 2015.

22. Solomon PJ, Margaret P, Rajendran R, Ramalingam R, Menezes GA, Shirley AS, Lee SJ, Seong MW, Park SS, Seol D and Seo SH: A case report and literature review of Fanconi Anemia (FA) diagnosed by genetic testing. Ital J Pediatr 41: 38, 2015.

23. Choi Y and Chan AP: PROVEAN web server: A tool to predict the functional effect of amino acid substitutions and indels. Bioinformatics 31: 2745-2747, 2015.

24. Nepal M, Che R, Ma C, Zhang J and Fei P: FANCD2 and DNA Damage. Int J Mol Sci 18: E1804, 2017.

25. MacMillan ML and Wagner JE: Haematopoeitic cell transplantation for Fonconi anaemia-when and how? Br I Haematol 149: $14-21,2010$

26. Ayas M: Hematopoietic cell transplantation in Fanconi Anemia and dyskeratosis congenita: A minireview. Hematol Oncol Stem Cell Ther 10: 285-289, 2017.

27. Guardiola P, Pasquini R, Dokal I, Ortega JJ, van Weel-Sipman M, Marsh JC, Ball SE, Locatelli F, Vermylen C, Skinner R, et al: Outcome of 69 allogeneic stem cell transplantations for Fanconi Anemia using HLA-matched unrelated donors: A study on behalf of the European Group for Blood and Marrow Transplantation. Blood 95: 422-429, 2000.

28. Blanche P; Diagnosis evaluation of FA[M]//Mary EE, Dave F, Lynn F, et al: Fanconi Anemia: Guidelines for diagnosis and management. 3rd ed. Eugene: Fanconi Anemia Res Funf Inc, 2008: $34-53$.

29. Końca K, Lankoff A, Banasik A, Lisowska H, Kuszewski T, Góźdź S, Koza Z and Wojcik A: A cross-platform public domain PC image-ayalysis program for the comet assay. Mutat Res 534, 15-20, 2003 . 